\title{
Assessment of Health-Related Quality of Life in Children with Rheumatic Heart Disease at Upper Egypt
}

Asmaa Mohammed Ismail, Hanan Mohammed Abd El-Moneim, Asmaa Ashraf Mohammed*

Department of Pediatrics, Faculty of Medicine, Aswan University, Aswan, Egypt

*Corresponding author: Asmaa Ashraf Mohammed, Mobile: (+20) 01127427797, E-Mail: simsma_24@yahoo.com

\begin{abstract}
Background: Many published literatures suggested that RHD can cause stress and anxiety in patients, with increased risks of evolving physical and psychosocial impairments. These include not only pain and fatigue but also difficulties with activities of daily living.

Objective: The study aimed to evaluate the HRQOL in children with RHD and delineate the HRQOL domains in various clinical subtypes of RHD.

Patients and methods: The present study was a cross-sectional, comparative, study that was conducted on 120 RHD patients who were recruited from Upper Egypt University Hospitals (Aswan, Assiut, and Qena hospitals) for six months. Also, 120 age and sex-matched healthy volunteers were included as the control group.

Results: In our cohort, the most common presentation of the included patients was pancarditis with arthritis (33.3\%), followed by polyarthritis (31.7\%), and fever with pan carditis (20\%). One-third of the patients had a history of one hospital admission, and $26.7 \%$ had a history of two or more admission. Besides, $65 \%$ had swollen, redness, hotness, limitation of movement in the lower limb. All patients were on long-acting penicillin and $10.8 \%$ had a history of cardiac surgery. In the present study, the mean serum CRP level was notably high in patients with RHD (mean 114.63 $\pm 113.5 \mathrm{mg} / \mathrm{dL}$ ).

Conclusion: Rheumatic heart disease imposed a considerable burden on the quality of life of the Egyptian pediatric population. In our study, we found that Egyptian patients with rheumatic heart disease had significant impairments on the physical, emotional, social, and school functioning, as compared to their counterpart healthy population.
\end{abstract}

Keywords: Health-related Quality of Life, Children, Rheumatic Heart Diseases, Upper Egypt.

\section{INTRODUCTION}

Acute Rheumatic Fever (ARF) is a postinfectious, non-suppurative sequel of pharyngeal infection with streptococcal pyogens, or group a betahemolytic streptococcus. Rheumatic fever occurs in 3$4 \%$ of the untreated group a streptococcal pharyngitis. Devastating complications of Rheumatic Heart Disease (RHD) include severe valve regurgitation, heart failure, strokes, and infective endocarditis, usually affecting both younger schools going and economically active, childbearing members of society ${ }^{(\mathbf{1})}$.

Many echocardiographic screening studies put the prevalence of RHD at 8-57 out of 1000 children meaning that the true prevalence may rest closer to $62-$ 78 million individuals worldwide with up to 1.4 million deaths each year. RHD has a high prevalence in Egypt. Although a high proportion of the abnormalities originally detected persisted at follow-up, both progression and regression of valve lesions were demonstrated ${ }^{(2)}$.

Quality of life (QoL) has received increased attention within medical and psychological research and practice Traditionally, psychological well-being has often been conceptualized in the negative sense as the absence of mental illness ${ }^{(3)}$.

The American Heart Association (AHA) included health-related Quality of life (HRQoL) evaluation as a strategic treatment-impact goal for cardiovascular health ${ }^{(4)}$ AHA defined HRQoL as the "discrepancy between actual and desired functional status and overall impact of health on well-being" (5).
The US Food and Drug Administration (FDA) released specific guidance for the development and validation of QOL measures that could be suitable for regulatory purposes. Further developed by FDA authors in 2016, the recommended QOL components for drug labeling purposes include disease-related symptoms, physical function, and patient-reported adverse events (6).

Rheumatic Heart Disease accounting for the greatest cardiovascular-related loss of disabilityadjusted life-years among 10-14-year old (516.6/100,000 individuals) and the second-highest number among children aged 5-9 years old $\left(362 / 100,000\right.$ individuals) ${ }^{(7)}$.

Quality of life in patients with chronic diseases is multifactorial conditioned. To some degree, quality of life is dependent upon the intensity and duration of its symptoms. Symptoms are influenced by activity, form location, and extent of disease. Psychological and social factors play a significant role, too ${ }^{(8)}$.

A recent study of children in Gulu, Uganda showed that being diagnosed with latent RHD was associated with the reduced physical and emotional quality of life scores even when patients were clinically asymptomatic ${ }^{(9)}$.

Also, Scheel $\boldsymbol{e t}$ al. ${ }^{\left({ }^{10)}\right.}$ said that as early RHD detection efforts expand, it is critically important to diminish the negative psychological impact associated with a positive screening result. To our knowledge, this is the first time a support group was studied amongst pediatric patients with RHD. Here, we examine the 
impact of a support group on RHD knowledge, healthrelated quality of life (HRQOL), and social support for children diagnosed with RHD.

\section{AIM OF THE WORK}

The study aimed to evaluate the HRQOL in children with RHD and delineate the HRQOL domains in various clinical subtypes of RHD. The primary objective was to assess the QOL in children between 3 and 18 years of age with RHD using the Pediatric Quality of Life Inventory (PedsQL ${ }^{\text {TM }} 4.0$ Generic Core scales) in relation to controls. The secondary objective was to evaluate the physical, emotional, and social domains affected in various clinical phenotypes of pediatric RHD.

\section{PATIENTS AND METHODS}

The present study was a cross-sectional, comparative, study that was conducted on 120 RHD patients who were recruited from Upper Egypt University Hospitals (Aswan, Assiut, and Qena hospitals) over six months. Also, 120 age and sexmatched healthy volunteers were included as a control group.

Studied populations were divided into two groups: Group I: Which included children with RHD and Group II: Which included age and sex-matched healthy volunteers.

Ethical Statement: We confirm that the present study was run in concordance with international ethical standards and applicable local regulatory guidelines. Written informed consent was obtained from the parents of every eligible patient. Parents were informed about the study objectives, methodology, risk, and benefit. The study's protocol was reviewed and approved by the institutional review board (IRB), ethics committee, or audit department of Faculty of Medicine, Aswan University.

Inclusion criteria: Children with ages of 3 to 18 -yearold. Children who were both mentally and educationally capable of answering the questionnaire. Case group: included children with RHD as diagnosed by the 2015 Jones criteria ${ }^{(11}$ while the control group: children did not have any clinical or biochemical features of RHD.

Exclusion criteria: Patients with chronic and painful health conditions like cancer, spine injury, and psychiatric morbidity. Patients who are not capable of answering the questionnaire either by them themselves or their parents. Patients with a history of congenital or non-RHDs.

Sample Size and Sampling: We utilized a nonprobability consecutive sampling technique. A total of 120 RHD children and a similar number of healthy controls were determined to be included in the present study.

Data Collection: All included patients were interviewed and data were collected in the form of a structured questionnaire (was filled from parents or caregivers of the included children) which includes the following data:

Full history taking: Child sex. Age. Residency. Consanguinity of parents.1 Family history of RHD. History of any other disease.

In addition to the Age of onset of RHD. Presenting symptoms (polyarthritis, polyarthralgia). History of hospital admission. History of long-acting penicillin administration. History of previous cardiac surgery.

Full physical examination, some anthropometric measurements (weight \& height).

1. Laboratory investigations included Complete blood count. Erythrocyte sedimentation rate (ESR). Creactive Protein (CRP). Antistreptolysin $\mathrm{O}$ titer (ASOT).

2. Imaging included Chest X-ray. Echocardiographic findings. Electrocardiogram (ECG).

3. Pediatric Quality of Life Inventory (PedsQLTM 4.0 Generic Core scales): The PedsQLTM 4-0 Generic Core Scales were designed to measure the core physical, mental, and social health dimensions as delineated by the World Health Organization ${ }^{(12)}$, as well as role (school) functioning. A previous report described the reliability and validity of the 23 items. pedsQLTM 4-0 Generic Core Scales in 1677 healthy, acutely ill, and chronically ill children ${ }^{(13)}$.

Study's Outcomes: The primary objective was to assess the QOL in children between 3 and 18 years of age with RHD using PedsQL ${ }^{\text {TM }} 4.0$ Generic Core scales in relation to controls. The secondary objective was to evaluate the physical, emotional, and social domains affected in various clinical phenotypes of pediatric RHD.

\section{Statistical Analysis}

Using Excel spreadsheet was established for the entry of data. We used validation checks on numerical variables and an option-based data entry method for categorical variables to reduce potential errors. The analyses were carried with SPSS software (Statistical Package for the Social Sciences, version 24, SSPS Inc, Chicago, IL, USA). The normality of the data was assessed using the Shapiro-Wilk Test. Numerical data were described as mean \pm SD if normally distributed, or median and interquartile range [IQR] if not normally distributed. Frequency tables with percentages were used for categorical variables. Independent Student t-test and paired t-test were used to compare parametric quantitative variables; while Mann-Whitney tests and Wilcoxon matched-pairs tests were used to compare non-parametric quantitative variables. Chi-square test or McNamara-Bowker tests were used to analyze categorical variables. Multilinear logistic regression was undertaken to assess the predictors of mortality. A p-value $<0.05$ is considered statistically significant. 
RESULTS

Table 1: The demographic characteristics of the included patients and controls

\begin{tabular}{|c|c|c|c|}
\hline Variables & $\begin{array}{c}\text { RHD } \\
(\mathrm{N}=120)\end{array}$ & $\begin{array}{c}\text { Control group } \\
(\mathbf{N}=120)\end{array}$ & P-value \\
\hline \multicolumn{4}{|c|}{ Age in years } \\
\hline Mean \pm SD & $9.77 \pm 2.3$ & $10.17 \pm 2.4$ & \multirow[t]{2}{*}{0.512} \\
\hline Median (range) & $9.7(7-14)$ & $9.5(7-14)$ & \\
\hline \multicolumn{4}{|c|}{ Gender, No. (\%) } \\
\hline Male & $72(60 \%)$ & $68(56.7 \%)$ & \multirow[t]{2}{*}{0.5} \\
\hline Female & $48(40 \%)$ & $52(43.3 \%)$ & \\
\hline \multicolumn{4}{|c|}{ Residency, No. (\%) } \\
\hline Assiut & $40(33.3 \%)$ & $48(40 \%)$ & \multirow{3}{*}{0.87} \\
\hline Aswan & $48(40 \%)$ & $36(30 \%)$ & \\
\hline Qena & $32(26.7 \%)$ & $36(30 \%)$ & \\
\hline \multicolumn{4}{|c|}{ Consanguinity, No. (\%) } \\
\hline Yes & $56(46.7 \%)$ & $44(36.7 \%)$ & \multirow[t]{2}{*}{0.602} \\
\hline No & $64(53.3 \%)$ & $76(67.3 \%)$ & \\
\hline \multicolumn{4}{|c|}{ Family history, No. (\%) } \\
\hline Yes & $26(21.6 \%)$ & 0 & \multirow{2}{*}{0.001} \\
\hline No & $94(78.4 \%)$ & $120(100 \%)$ & \\
\hline
\end{tabular}

$*$ Data are presented as mean $\pm \mathrm{SD}$, Median (Range), or number $(\%)$

Table 1 shows the demographic characteristics of the included subjects. There was a statistically significant difference between RHD patients and the control group in terms of family history ( $p=0.001)$, patients with RHD were more likely to have a positive family history. In contrary, there were no statistically significant differences between RHD patients and control group in term of age $(\mathrm{p}=0.512)$, gender $(\mathrm{p}=0.5)$, residency $(\mathrm{p}=0.87)$, and consanguinity $(\mathrm{p}$ $=0.602$ ).

Table 2: The disease characteristics of the included patients

\begin{tabular}{|c|c|}
\hline Variables & RHD (N =120) \\
\hline \multicolumn{2}{|l|}{ Age at onset } \\
\hline - $\quad$ Mean \pm SD & $7.2 \pm 2.3$ \\
\hline \multicolumn{2}{|l|}{ Presentation, No. (\%) } \\
\hline - Chorea & $6(5 \%)$ \\
\hline - $\quad$ Fever, pancarditis & $24(20 \%)$ \\
\hline - $\quad$ Pancarditis & $12(10 \%)$ \\
\hline - $\quad$ Pancarditis, arthritis & $40(33.3 \%)$ \\
\hline - $\quad$ Polyarthritis & $38(31.7 \%)$ \\
\hline \multicolumn{2}{|c|}{ History of hospital admission, No. (\%) } \\
\hline - $\quad$ No & $48(40 \%)$ \\
\hline - $\quad 1$ time & $40(33.3 \%)$ \\
\hline - $\quad 2$ times & $12(10 \%)$ \\
\hline - $\quad 3$ times & $20(16.7 \%)$ \\
\hline \multicolumn{2}{|l|}{ Treatment, No. (\%) } \\
\hline On long-acting penicillin & $120(100 \%)$ \\
\hline \multicolumn{2}{|c|}{ History of cardiac surgery, No. (\%) } \\
\hline - Yes & $13(10.8 \%)$ \\
\hline$-\quad$ No & $107(89.2 \%)$ \\
\hline
\end{tabular}

*Data are presented as mean $\pm \mathrm{SD}$, Median (Range), or number (\%)

Table 2 shows the disease characteristics of the included subjects. The mean age at onset was $7.2 \pm 2.3$ years old. The most common presentation of the included patients was pancarditis with arthritis (33.3\%), followed by polyarthritis $(31.7 \%)$, and fever with pancarditis $(20 \%)$. One-third of the patients had a history of one hospital admission, and $26.7 \%$ had a history of two or more admission. All patients were on long-acting penicillin and $10.8 \%$ had a history of cardiac surgery. 
Table 3: Findings of the General Examination in patients

\begin{tabular}{|c|c|c|}
\hline \multirow{2}{*}{ Variables, mean \pm SD } & \multicolumn{2}{|c|}{ RHD (N =120) } \\
\hline & mean \pm SD & Median (range) \\
\hline Weight (kg) & $28.65 \pm 4.9$ & $28(22-36)$ \\
\hline Height $(\mathrm{cm})$ & $128.5 \pm 6.3$ & $127(118-140)$ \\
\hline $\operatorname{BMI}\left(\mathrm{Kg} / \mathrm{m}^{2}\right)$ & $17.8 \pm 2.25$ & $18(17.2-22.5)$ \\
\hline Temperature (c) & $38.1 \pm 0.5$ & $38(37.5-39)$ \\
\hline Heart Rate (beats/minutes) & $103.3 \pm 25.3$ & $97.5(70-140)$ \\
\hline Respiratory rate (per minutes) & $33.27 \pm 8.3$ & $29(25-45)$ \\
\hline $\begin{array}{l}\text { General Condition, No. (\%) } \\
\text { Fair } \\
\text { Bad }\end{array}$ & \multicolumn{2}{|c|}{$\begin{array}{l}68(66.7 \%) \\
40(33.3 \%)\end{array}$} \\
\hline $\begin{array}{l}\text { LL, No. (\%): } \\
\text { Swollen, redness, hotness, limitation of movement }\end{array}$ & \multicolumn{2}{|c|}{$78(65 \%)$} \\
\hline
\end{tabular}

*Data are presented as mean \pm SD and median (range)

Table 3 shows the findings of the clinical examination of the included subjects. The mean weight and height were $28.65 \pm 4.9 \mathrm{Kg}$ and $128.5 \pm 6.3 \mathrm{~cm}$, respectively; while the mean BMI was $17.8 \pm 2.25 \mathrm{Kg} / \mathrm{m}^{2}$. The mean heart and respiratory rates were $103.3 \pm 25.3$ beats/min and $33.27 \pm 8.3$ per minute, respectively. One-third of the patients had bad general condition and $65 \%$ had swollen, redness, hotness, limitation of movement in the lower limb.

Table 4: Findings of the systematic examination

\begin{tabular}{||l||ll||c||}
\hline \hline \multicolumn{1}{|c||}{} & \multicolumn{1}{|c||}{ Variables } & RHD (N =120) \\
\hline \hline Chest, No. (\%) & - & Normal vesicular sound & $120(100 \%)$ \\
\hline \multirow{2}{*}{ CVS, No. (\%) } & - & Pan systolic murmur & $120(100 \%)$ \\
& - & Early diastolic murmur & $68(56.7 \%)$ \\
\hline \hline GIT, No. (\%) & - & No organomegaly & $120(100 \%)$ \\
\hline
\end{tabular}

*Data are presented as number $(\%)$

Table 4 shows the findings of the systematic examination of the included subjects. All patients had a pansystolic murmur and $56.7 \%$ had an early diastolic murmur. None of the patients had organomegaly.

Table 5: The Laboratory findings of the included patients

\begin{tabular}{||l||c|c||}
\hline & Variables & RHD (N =120) \\
\hline \hline Hemoglobin (g/dL) & - Mean \pm SD & $9.62 \pm 0.6$ \\
\hline \hline CRP (mg/dL) & - Mean \pm SD & $114.63 \pm 13.5$ \\
\hline \hline ASOT & - Mean \pm SD & $407.13 \pm 13.2$ \\
\hline
\end{tabular}

*Data are presented as mean $\pm \mathrm{SD}$, Median (Range), or number (\%).

Table 5 shows the laboratory findings of the included subjects. The mean hemoglobin level was $9.62 \pm 0.6 \mathrm{~g} / \mathrm{dL}$ and the mean CRP was $114.63 \pm 113.5 \mathrm{mg} / \mathrm{dL}$. The mean ASOT was 407.13 \pm 113.2 .

Table 6: Imaging Findings in patients

\begin{tabular}{|c|c|c|}
\hline & Variables & RHD (N =120) \\
\hline X-ray, No. (\%) & - $\quad$ Cardiomegaly & $120(100 \%)$ \\
\hline \multirow{3}{*}{ ECG, No. (\%) } & - $\quad$ Inversion of T1, partial A-V block & $24(20 \%)$ \\
\hline & - $\quad$ Partial A-V block & $32(26.7 \%)$ \\
\hline & - $\quad$ Prolonged P-R interval & $78(65 \%)$ \\
\hline \multirow{5}{*}{ Echo, No. (\%) } & Mitral regurge & $44(36.7 \%)$ \\
\hline & - $\quad$ Mitral regurge, aortic regurge & $28(23.3 \%)$ \\
\hline & - $\quad$ Mitral regurge, tricuspid regurge & $12(10 \%)$ \\
\hline & - $\quad$ Severe mitral regurge, severe aortic regurge & $24(20 \%)$ \\
\hline & - $\quad$ Severe mitral regurge, trace aortic regurge & $12(10 \%)$ \\
\hline
\end{tabular}

*Data are presented as number $(\%)$

Table 6 shows the imaging findings of the included subjects. All patients had cardiomegaly on X-ray and $65 \%$ had prolonged P-R interval on ECG. The ECHO showed that 36.7\% of the patients had isolated mitral regurge, $23.3 \%$ had mitral and aortic regurge, $10 \%$ had mitral and tricuspid regurge, $20 \%$ had severe mitral and aortic regurge, and $10 \%$ had severe mitral regurge and trace aortic regurge. 
Table 7: The physical health domain of the Peds QL 4.0

\begin{tabular}{|c|c|c|c|}
\hline Variables & $\begin{array}{c}\text { RHD } \\
(\mathbf{N}=120)\end{array}$ & $\begin{array}{c}\text { Control group } \\
(N=120)\end{array}$ & P-value \\
\hline $\begin{array}{ll} & \text { Walking more than one block, No. } \\
& (\boldsymbol{\%}) \\
- & \text { Never (0) } \\
- & \text { Almost Never (1) } \\
- & \text { Sometimes (2) } \\
- & \text { Often (3) } \\
- & \text { Almost always (4) } \\
\end{array}$ & $\begin{array}{c}0 \\
52(43.3 \%) \\
16(13.3 \%) \\
32(26.7 \%) \\
20(16.7 \%) \\
\end{array}$ & $\begin{array}{c}64(53.3 \%) \\
40(33.3 \%) \\
8(6.7 \%) \\
8(6.7 \%) \\
0 \\
\end{array}$ & $<0.001$ \\
\hline $\begin{array}{ll} & \text { Running, No. (\%) } \\
- & \text { Never (0) } \\
- & \text { Almost Never (1) } \\
- & \text { Sometimes (2) } \\
- & \text { Often (3) } \\
- & \text { Almost always (4) } \\
\end{array}$ & $\begin{aligned} & 8(6.7 \%) \\
& 52(43.3 \%) \\
& 8(6.7 \%) \\
& 32(26.7 \%) \\
& 20(16.7 \%) \\
&\end{aligned}$ & $\begin{array}{c}44(36.7 \%) \\
56(46.7 \%) \\
12(10 \%) \\
8(6.7 \%) \\
0 \\
\end{array}$ & 0.005 \\
\hline $\begin{array}{ll} & \text { Participating in sports activity or } \\
& \text { exercise, No. (\%) } \\
- & \text { Never }(0) \\
- & \text { Almost Never (1) } \\
- & \text { Sometimes (2) } \\
- & \text { Often (3) } \\
- & \text { Almost always (4) } \\
\end{array}$ & $\begin{array}{c}0 \\
28(23.3 \%) \\
20(16.7 \%) \\
52(43.3 \%) \\
16(13.3 \%) \\
\end{array}$ & $\begin{array}{c}48(40 \%) \\
52(43.3 \%) \\
12(10 \%) \\
8(6.7 \%) \\
0 \\
\end{array}$ & $<0.001$ \\
\hline $\begin{array}{ll} & \text { Lifting something heavy, No. (\%) } \\
- & \text { Never (0) } \\
- & \text { Almost Never (1) } \\
- & \text { Sometimes (2) } \\
- & \text { Often (3) } \\
- & \text { Almost always (4) } \\
\end{array}$ & $\begin{array}{r}4(3.3 \%) \\
24(20 \%) \\
32(26.7 \%) \\
32(26.7 \%) \\
28(23.3 \%) \\
\end{array}$ & $\begin{array}{c}64(53.3 \%) \\
40(33.3 \%) \\
12(10 \%) \\
8(6.7 \%) \\
0 \\
\end{array}$ & $<0.001$ \\
\hline $\begin{array}{ll} & \text { Taking a bath or shower, No. (\%) } \\
- & \text { Never (0) } \\
- & \text { Almost Never (1) } \\
- & \text { Sometimes (2) } \\
- & \text { Often (3) } \\
- & \text { Almost always (4) } \\
\end{array}$ & $\begin{array}{c}0 \\
20(16.7 \%) \\
40(33.3 \%) \\
40(33.3 \%) \\
20(16.7 \%) \\
\end{array}$ & $\begin{array}{c}108(90 \%) \\
12(10 \%) \\
0 \\
0 \\
0\end{array}$ & $<0.001$ \\
\hline $\begin{array}{ll} & \text { Doing chores, No. (\%) } \\
\text { - } & \text { Never (0) } \\
- & \text { Almost Never (1) } \\
- & \text { Sometimes (2) } \\
- & \text { Often (3) } \\
- & \text { Almost always (4) } \\
\end{array}$ & $\begin{array}{c}0 \\
40(33.3 \%) \\
60(50 \%) \\
20(16.7 \%) \\
0 \\
\end{array}$ & $\begin{array}{c}112(93.3 \%) \\
8(6.7 \%) \\
0 \\
0 \\
0 \\
\end{array}$ & $<0.001$ \\
\hline $\begin{array}{ll} & \text { Having hurts or aches, No. (\%) } \\
- & \text { Never (0) } \\
- & \text { Almost Never (1) } \\
- & \text { Sometimes (2) } \\
- & \text { Often (3) } \\
- & \text { Almost always (4) }\end{array}$ & $\begin{array}{c}0 \\
20(16.7 \%) \\
16(13.3 \%) \\
48(40 \%) \\
36(30 \%)\end{array}$ & $\begin{array}{c}112(93.3 \%) \\
8(6.7 \%) \\
0 \\
0 \\
0\end{array}$ & $<0.001$ \\
\hline
\end{tabular}

*Data are presented as mean $\pm \mathrm{SD}$, Median (Range), or number (\%)

Table 7 shows the physical health domain of Peds QL 4.0. Patients with RHD were more likely to have limitations in walking more than one block $(\mathrm{p}<0.001)$, running $(\mathrm{p}<0.001)$, participating in sports activity or exercise ( $\mathrm{p}<0.001)$, lifting something heavy ( $\mathrm{p}<0.001)$, taking a bath or shower $(\mathrm{p}<0.001)$, doing chores $(\mathrm{p}<0.001)$. Also, patients with RHD were more likely to have hurts or aches $(\mathrm{p}<0.001)$. 
Table 8: The emotional functioning domain of the PedsQL 4.0

\begin{tabular}{|c|c|c|c|}
\hline Variables & $\begin{array}{c}\text { RHD } \\
(\mathrm{N}=120)\end{array}$ & $\begin{array}{l}\text { Control group } \\
(\mathbf{N}=120)\end{array}$ & P-value \\
\hline $\begin{array}{ll} & \text { Feeling afraid or scared, No. (\%) } \\
- & \text { Never (0) } \\
- & \text { Almost Never (1) } \\
- & \text { Sometimes (2) } \\
- & \text { Often (3) } \\
- & \text { Almost always (4) }\end{array}$ & $\begin{array}{c}0 \\
20(16.7 \%) \\
40(33.3 \%) \\
40(33.3 \%) \\
20(16.7 \%) \\
\end{array}$ & $\begin{array}{c}48(40 \%) \\
52(43.3 \%) \\
12(10 \%) \\
8(6.7 \%) \\
0\end{array}$ & $<0.001$ \\
\hline $\begin{array}{ll} & \text { Feeling sad or blue, No. (\%) } \\
- & \text { Never (0) } \\
- & \text { Almost Never (1) } \\
- & \text { Sometimes (2) } \\
- & \text { Often (3) } \\
- & \text { Almost always (4) } \\
\end{array}$ & $\begin{array}{c}0 \\
0 \\
40(33.3 \%) \\
60(50 \%) \\
20(16.7 \%) \\
\end{array}$ & $\begin{array}{c}108(90 \%) \\
12(10 \%) \\
0 \\
0 \\
0 \\
\end{array}$ & $<0.001$ \\
\hline $\begin{array}{ll} & \text { Feeling angry, No. (\%) } \\
- & \text { Never (0) } \\
- & \text { Almost Never (1) } \\
- & \text { Sometimes (2) } \\
- & \text { Often (3) } \\
- & \text { Almost always (4) } \\
\end{array}$ & $\begin{array}{c}0 \\
20(16.7 \%) \\
16(13.3 \%) \\
48(40 \%) \\
36(30 \%) \\
\end{array}$ & $\begin{array}{c}120(100 \%) \\
0 \\
0 \\
0 \\
0 \\
\end{array}$ & $<0.001$ \\
\hline $\begin{array}{ll} & \text { Trouble sleeping, No. (\%) } \\
- & \text { Never (0) } \\
- & \text { Almost Never (1) } \\
- & \text { Sometimes (2) } \\
- & \text { Often (3) } \\
- & \text { Almost always (4) } \\
\end{array}$ & $\begin{array}{c}0 \\
32(23.3 \%) \\
44(36.7 \%) \\
36(30 \%) \\
12(10 \%) \\
\end{array}$ & $\begin{array}{c}88(73.3 \%) \\
20(16.7 \%) \\
12(10 \%) \\
0 \\
0 \\
\end{array}$ & $<0.001$ \\
\hline $\begin{array}{ll} & \text { Worrying about what will happen, } \\
& \text { No. }(\%) \\
- & \text { Never (0) } \\
- & \text { Almost Never (1) } \\
- & \text { Sometimes (2) } \\
- & \text { Often (3) } \\
- & \text { Almost always (4) }\end{array}$ & $\begin{array}{c}0 \\
16(13.3 \%) \\
28(23.3 \%) \\
56(46.7 \%) \\
20(16.7 \%) \\
\end{array}$ & $\begin{array}{c}120(100 \%) \\
0 \\
0 \\
0 \\
0\end{array}$ & $<0.001$ \\
\hline
\end{tabular}

$*$ Data are presented as mean \pm SD, Median (Range), or number $(\%)$

Table 8 shows the emotional domain of Peds QL 4.0. Patients with RHD were more likely to have to feel afraid or scared ( $p<0.001)$, feeling sad or blue ( $p<0.001)$, feeling angry ( $<<0.001)$, trouble sleeping $(\mathrm{p}<0.001)$, and worrying about what will happen $(\mathrm{p}<0.001)$. 
Table 9: The social functioning domain of the PedsQL 4.0

\begin{tabular}{|c|c|c|c|}
\hline Variables & $\begin{array}{c}\text { RHD } \\
(\mathbf{N}=120) \\
\end{array}$ & $\begin{array}{c}\text { Control group } \\
(\mathbf{N}=120) \\
\end{array}$ & $\overline{P \text { P-value }}$ \\
\hline $\begin{array}{ll} & \text { Getting along with other children, } \\
& \text { No. (\%) } \\
- & \text { Never (0) } \\
- & \text { Almost Never (1) } \\
- & \text { Sometimes (2) } \\
- & \text { Often (3) } \\
- & \text { Almost always (4) } \\
\end{array}$ & $\begin{array}{c}14(11.7 \%) \\
46(38.3 \%) \\
59(49.2 \%) \\
1(0.8 \%) \\
0\end{array}$ & $\begin{array}{c}0 \\
2(1.6 \%) \\
17(14.2 \%) \\
57(47.5 \%) \\
44(36.7 \%)\end{array}$ & $<0.001$ \\
\hline $\begin{array}{ll} & \text { Other kids not wanting to be his or } \\
\text { her friend, No. }(\boldsymbol{\%}) \\
- & \text { Never (0) } \\
- & \text { Almost Never (1) } \\
- & \text { Sometimes (2) } \\
- & \text { Often (3) } \\
- & \text { Almost always (4) } \\
\end{array}$ & $\begin{array}{c}5(4.2 \%) \\
15(12.5 \%) \\
40(33.3 \%) \\
60(50 \%) \\
0 \\
\end{array}$ & $\begin{array}{c}99(82.5 \%) \\
7(5.8 \%) \\
14(11.7 \%) \\
0 \\
0 \\
\end{array}$ & $<0.001$ \\
\hline $\begin{array}{ll} & \text { Getting teased by other children, No. } \\
(\boldsymbol{\%}) \\
- & \text { Never (0) } \\
- & \text { Almost Never (1) } \\
- & \text { Sometimes (2) } \\
- & \text { Often (3) } \\
- & \text { Almost always (4) } \\
\end{array}$ & $\begin{array}{c}0 \\
20(16.7 \%) \\
16(13.3 \%) \\
48(40 \%) \\
36(30 \%) \\
\end{array}$ & $\begin{array}{c}78(65 \%) \\
12(10 \%) \\
30(25 \%) \\
0 \\
0 \\
\end{array}$ & $<0.001$ \\
\hline $\begin{array}{ll} & \begin{array}{l}\text { Not able to do things that other } \\
\text { children his or her age can do, No. } \\
(\%)\end{array} \\
- & \text { Never (0) } \\
- & \text { Almost Never (1) } \\
- & \text { Sometimes (2) } \\
- & \text { Often (3) } \\
- & \text { Almost always (4) }\end{array}$ & $\begin{array}{c}8(6.7 \%) \\
20(26.7 \%) \\
16(13.3 \%) \\
32(26.7 \%) \\
44(36.7 \%)\end{array}$ & $\begin{array}{c}88(73.3 \%) \\
20(16.7 \%) \\
12(10 \%) \\
0 \\
0\end{array}$ & $<0.001$ \\
\hline $\begin{array}{ll} & \begin{array}{l}\text { Keeping up when playing with other } \\
\text { children, No. }(\%)\end{array} \\
- & \text { Never (0) } \\
- & \text { Almost Never (1) } \\
- & \text { Sometimes (2) } \\
- & \text { Often (3) } \\
- & \text { Almost always (4) }\end{array}$ & $\begin{array}{c}13(10.8 \%) \\
56(46.7 \%) \\
42(35 \%) \\
10(13.3 \%) \\
0\end{array}$ & $\begin{array}{c}0 \\
0 \\
15(12.5 \%) \\
86(71.7 \%) \\
19(15.7 \%)\end{array}$ & $<0.001$ \\
\hline
\end{tabular}

*Data are presented as mean $\pm \mathrm{SD}$, Median (Range), or number $(\%)$

Table 9 shows the social functioning domain of Peds QL 4.0. Patients with RHD were more likely to have troubles in getting along with other children ( $\mathrm{p}<0.001$ ), making friends ( $\mathrm{p}<0.001$ ), doing things that other children his or her age can do ( $\mathrm{p}<0.001)$, and keeping up when playing with other children ( $<<0.001)$. In addition, patients with RHD were more likely to be teased by other children ( $\mathrm{p}<0.001)$. 
Table 10: The school functioning domain of the PedsQL 4.0

\begin{tabular}{|c|c|c|c|}
\hline Variables & $\begin{array}{c}\text { RHD } \\
(\mathbf{N}=120) \\
\end{array}$ & $\begin{array}{c}\begin{array}{c}\text { Control group } \\
(\mathrm{N}=120)\end{array} \\
\end{array}$ & P-value \\
\hline $\begin{array}{ll} & \text { Paying attention in class, No. (\%) } \\
- & \text { Never (0) } \\
- & \text { Almost Never (1) } \\
- & \text { Sometimes (2) } \\
- & \text { Often (3) } \\
- & \text { Almost always (4) }\end{array}$ & $\begin{array}{l}13(10.8 \%) \\
56(46.7 \%) \\
42(35 \%) \\
10(13.3 \%) \\
0\end{array}$ & $\begin{array}{c}0 \\
2(1.6 \%) \\
17(14.2 \%) \\
57(47.5 \%) \\
44(36.7 \%)\end{array}$ & $<0.001$ \\
\hline $\begin{array}{ll} & \text { Forgetting things, No. (\%) } \\
\text { - } & \text { Never (0) } \\
- & \text { Almost Never (1) } \\
- & \text { Sometimes (2) } \\
- & \text { Often (3) } \\
- & \text { Almost always (4) } \\
\end{array}$ & $\begin{array}{c}0 \\
20(16.7 \%) \\
16(13.3 \%) \\
48(40 \%) \\
36(30 \%) \\
\end{array}$ & $\begin{array}{l}78(65 \%) \\
12(10 \%) \\
30(25 \%) \\
0 \\
0 \\
\end{array}$ & $<0.001$ \\
\hline $\begin{array}{ll} & \text { Keeping up with schoolwork, No. } \\
& (\boldsymbol{\%}) \\
- & \text { Never (0) } \\
- & \text { Almost Never (1) } \\
- & \text { Sometimes (2) } \\
- & \text { Often (3) } \\
- & \text { Almost always (4) } \\
\end{array}$ & $\begin{array}{l}13(10.8 \%) \\
56(46.7 \%) \\
42(35 \%) \\
10(13.3 \%) \\
0 \\
\end{array}$ & $\begin{array}{c}0 \\
0 \\
15(12.5 \%) \\
86(71.7 \%) \\
19(15.7 \%) \\
\end{array}$ & $<0.001$ \\
\hline $\begin{array}{ll} & \text { Missing school because of not feeling } \\
\text { well, No. (\%) } \\
-\quad \text { Never (0) } \\
- & \text { Almost Never (1) } \\
- & \text { Sometimes (2) } \\
- & \text { Often (3) } \\
- & \text { Almost always (4) } \\
\end{array}$ & $\begin{array}{c}0 \\
20(16.7 \%) \\
16(13.3 \%) \\
48(40 \%) \\
36(30 \%) \\
\end{array}$ & $\begin{array}{c}68(56.7 \%) \\
22(18.3 \%) \\
30(25 \%) \\
0 \\
0 \\
\end{array}$ & $<0.001$ \\
\hline $\begin{array}{ll} & \text { Missing school to go to the doctor or } \\
\text { hospital, No. (\%) } \\
-\quad \text { Never (0) } \\
-\quad \text { Almost Never (1) } \\
-\quad \text { Sometimes (2) } \\
-\quad \text { Often (3) } \\
-\quad \text { Almost always (4) }\end{array}$ & $\begin{array}{l}4(3.3 \%) \\
24(20 \%) \\
32(26.7 \%) \\
32(26.7 \%) \\
28(23.3 \%)\end{array}$ & $\begin{array}{l}64(53.3 \%) \\
40(33.3 \%) \\
12(10 \%) \\
8(6.7 \%) \\
0\end{array}$ & $<0.001$ \\
\hline
\end{tabular}

$*$ Data are presented as mean \pm SD, Median (Range), or number (\%)

Table 10 shows the school functioning domain of Peds QL 4.0. Patients with RHD were more likely to have troubles in paying attention in class $(\mathrm{p}<0.001)$, forgetting things $(\mathrm{p}<0.001)$, keeping up with schoolwork $(\mathrm{p}<0.001)$, missing school because of not feeling well ( $\mathrm{p}<0.001)$, and missing school to go to the doctor or hospital $(\mathrm{p}<0.001)$.

\section{DISCUSSION}

The present study was a cross-sectional, comparative, study that was conducted on 120 RHD patients who were recruited from Upper Egypt University Hospitals (Aswan, Assiut, and Qena hospitals) over six months. Besides, 120 age and sexmatched healthy volunteers were included as the control group.

The currently published data showed that RHD mainly affects school-children aged between 5 and 15 years, with slight male predominance ${ }^{(14)}$. On the other hand, a positive family history of ARF/RHD and paternal consanguinity are significantly associated with the occurrence of RHD ${ }^{(15)}$.

In the present study, the mean age of the included patients was $9.77 \pm 2.3$ and $60 \%$ of them were males. There was a statistically significant difference between RHD patients and the control group in terms of family history ( $\mathrm{p}=0.001$ ), patients with RHD were more likely to have a positive family history. On the contrary, there were no statistically significant differences between RHD patients and the control group in terms of consanguinity ( $\mathrm{p}=0.602$ ).

In line with our findings, Paar et al. (16) performed an observational study to determine the prevalence of RHD in 3150 children, ages 5-15 in León, Nicaragua. The mean age of the included patients was $9.5 \pm 1.3$ and $66 \%$ of them were males.

Likewise, Animasahun et al. ${ }^{(17)}$ conducted a review of prospectively collected data of patients with rheumatic heart disease who had echocardiography done from April 2007-Dec 2016. A total of 324,676 patients were seen at the Paediatric Unit of LASUTH from 2007 to 2016, out of which 36 had Rheumatic 
heart disease. The mean age of patients was $9.12 \pm 2.75$ years with a male to female ratio of 1.6: 1 .

Also, Boyarchuk et al. ${ }^{(18)}$ investigated the role of demographic, social, and medical risk factors for the development of RHD in a low-risk area (Western Ukraine). Between 2007-2014, 132 subjects aged 8-17 years from three medical centers were enrolled in the study. Of them, 78 patients developed RHD. Children with a positive family history were found to be at significantly higher risk of development of RHD.

In our cohort, the most common presentation of the included patients was pancarditis with arthritis (33.3\%), followed by polyarthritis (31.7\%), and fever with pancarditis (20\%). One-third of the patients had a history of one hospital admission, and $26.7 \%$ had a history of two or more admission. Also, $65 \%$ had swollen, redness, hotness, limitation of movement in the lower limb. All patients were on long-acting penicillin and $10.8 \%$ had a history of cardiac surgery.

Similar to our findings, Borges et al. (19) conducted a cross-sectional study of patients consecutively seen in the Cardiology Ward at FUNDHACRE. From July 2003 to February 2004, 99 patients with RHD were assessed with a mean age was 9.1 years old. The most frequent clinical manifestations were carditis (69.7\%), arthritis (21.4\%), and chorea $(6.1 \%)$.

Sharma et al. ${ }^{(20)}$ conducted a hospital-based observational study at Shahid Gangalal National Heart Center where all children less than 15 years of age with confirmed echocardiographic diagnosis of RHD from June 2015 to May 2016. Two hundred and eleven children were diagnosed with RHD. The most common presentation was carditis $(69.7 \%)$ and arthritis $(21.4 \%)$.

It is well known that C-reactive protein (CRP) is increased in patients with acute rheumatic fever, but it is not known whether plasma levels increase in patients with chronic rheumatic valve disease ${ }^{(21)}$. In the present study, the mean serum CRP level was notably high in patients with RHD (mean 114.63 $\pm 113.5 \mathrm{mg} / \mathrm{dL}$ ).

In agreement with our findings, Gölbasi $\boldsymbol{e t}$ al . (21) aimed to determine the role of inflammation detected by CRP levels in the progression of chronic rheumatic valve disease. A total of 113 patients with chronic rheumatic valve disease and 102 healthy subjects, as a control group, were assessed. Levels of CRP were significantly higher in patients with chronic rheumatic heart disease than in healthy subjects.

Similarly, Attar et al. ${ }^{(22)}$ assessed the possible association between CRP level as an indicator of inflammation and RHD. Ninety patients with RHD and 90 healthy controls who had undergone complete echocardiographic examination were enrolled in this cross-sectional study. The mean plasma CRP level in the case group was significantly higher compared to its level in the control group. These findings can be attributed to the persistence of inflammation in the chronic phase in RHD.
In our cohort, the echocardiography showed that $36.7 \%$ of the patients had isolated mitral regurge, $23.3 \%$ had mitral and aortic regurge, $10 \%$ had mitral and tricuspid regurge, $20 \%$ had severe mitral and aortic regurge, and $10 \%$ had severe mitral regurge and trace aortic regurge.

(23) In agreement with our findings, Shrestha et al. echocardiography reports from patients diagnosed with RHD between June 1999 and February 2011. Among 10860 transthoracic echocardiography studies, 1055 females and 658 male patients were diagnosed with RHD. Mitral regurgitation was the most common valvular lesion across all age groups. The involvement of both the mitral and the aortic valve was observed in $49.8 \%$ of the patients.

Likewise, Manjunath et al. ${ }^{(24)}$ reported that the mitral valve was the most common involved valve in the rheumatic group, seen in $60.2 \%$ of the cases. Another $24.5 \%$ of cases had involvement of the aortic valve as well, making the overall involvement of the mitral valve in RHD to be $84.7 \%$. The aortic valve was involved in $29.1 \%$ of cases making it the second most common valve to be involved in RHD. The next most common to be involved was the Tricuspid valve (10.7\% of rheumatic cohort). Pulmonary was the least common valve to be involved in RHD $(0.04 \%)$.

Such findings reflect the pathological involvement of the cardiac valves in ARF with mitral being the most common and pulmonary, the least ${ }^{(25)}$.

The PedsQL (Pediatric Quality of Life Inventory) (Children's Hospital and Health Center, San Diego, California) is a modular instrument for measuring health-related quality of life (HRQOL) in children and adolescents ages 2 to 18 . The PedsQL 4.0 Generic Core Scales are multidimensional child selfreport and parent proxy-report scales developed as the generic core measure to be integrated with the PedsQL Disease-Specific Modules. The PedsQL 4.0 Generic Core Scales consist of 23 items applicable to healthy school and community populations, as well as pediatric populations with acute and chronic health conditions (26).

In the present study, the 4 PedsQL 4.0 Generic Core Scales (Physical, Emotional, Social, School) were administered to RHD children and healthy controls. In terms of the physical health domain of Peds QL 4.0, we found that patients with RHD were more likely to have limitations in walking more than one block ( $\mathrm{p}<0.001)$, running ( $\mathrm{p}<0.001)$, participating in sports activity or exercise ( $p<0.001)$, lifting something heavy ( $p<0.001$ ), taking a bath or shower ( $\mathrm{p}<0.001)$, doing chores ( $\mathrm{p}$ $<0.001)$. In addition, patients with RHD were more likely to have hurts or aches ( $p<0.001)$. There was a statistically significant difference between RHD patients and the control group in terms of physical health score $(\mathrm{p}<0.001)$.

In concordance with our findings, Riaz et al. ${ }^{(27)}$ conducted a case-control study to assess the quality of 
life in patients with RHD. This case-control study was conducted at the Gulab Devi Chest Hospital, Lahore, Pakistan, from October 2016 to March 2017. Of the 300 subjects, $150(50 \%)$ each were cases and controls. The patients had significantly reduced scores in all QoL aspects including general health $(\mathrm{p}<0.001)$, physical functioning $(\mathrm{p}<0.001)$.

Similarly, in Egypt, a sample of 400 parents of children with heart disease ( $32.5 \%$ with RHD) was compared with 400 parents of children with minor illness with an Arabic version of a standardized quality of life questionnaire (SF-36). The parents of children with heart disease reported that their children had significantly $(P<0.001)$ different HRQOL with a reduction in general health and physical functioning and with role limitations as a result of physical, emotional, and social problems ${ }^{(28)}$.

The impact of RHD on physical functionrelated quality of life can be explained by the fact that heart disease employs a significant impact on physical health from the medical point of view. Patients diagnosed with the disease experience severe difficulties due to complications caused by this disease such as atrial fibrillation, heart failure, and consequently often have problems in maintaining normal daily activities ${ }^{(29)}$.

However, the current published literature remains inconclusive regarding the impact of RHD on quality of life. In contrary to our findings, Essawy et al. (30) aimed to identify the health-related quality of life of school-age children with rheumatic fever. A convenient sample of 100 school-age children with RF and their mothers were selected from the outpatient clinic and inpatient pediatric cardiac departments of EL-Shatby Children University Hospital, Alexandria, Egypt. The majority (78.3\%) of school-age children with rheumatic fever had a neutral HRQOL and less than a quarter of them had high HRQOL. Only a small percent $(8.7 \%)$ of the studied subjects had poor HRQOL.

The difference between our findings and the abovementioned study can be explained by the fact that Essawy et al. ${ }^{(30)}$ included only patients with ARF. In addition, the lack of a matched control group may affect the validity of Essawy et al. ${ }^{(30)}$ conclusion.

Regarding the impact of RHD on psychological well-being, we found that patients with RHD were more likely to have to feel afraid or scared ( $p<0.001)$, feeling sad or blue $(\mathrm{p}<0.001)$, feeling angry $(\mathrm{p}<0.001)$, trouble sleeping ( $p<0.001)$, worrying about what will happen ( $p<0.001)$, have troubles in getting along with other children ( $p<0.001)$, making friends ( $p<0.001)$, doing things that other children his or her age can do ( $p$ $<0.001$ ), and keeping up when playing with other children ( $\mathrm{p}<0.001)$. There was a statistically significant difference between RHD patients and the control group in terms of emotional functioning score $(\mathrm{p}<0.001)$ and social functioning score $(\mathrm{p}<0.001)$.

In agreement with our findings, Riaz et al. ${ }^{(27)}$ reported that RHD patients had significantly reduced scores in all QoL aspects including emotional and social domains.

The low scores in the emotional and social dimensions in RHD can be explained by the fact that RHD patients suffer from their chronic illness. It was shown that adolescents with heart disease reported a higher level of anxiety and depression as compared to age-matched healthy controls ${ }^{(31)}$.

In terms of the school functioning domain of Peds QL 4.0. we found that patients with RHD were more likely to have troubles in paying attention in class ( $p<0.001$ ), forgetting things ( $p<0.001)$, keeping up with schoolwork ( $p<0.001$ ), missing school because of not feeling well ( $p<0.001$ ), and missing school to go to the doctor or hospital ( $\mathrm{p}<0.001)$. There was a statistically significant difference between RHD patients and the control group in terms of the school functioning domain ( $\mathrm{p}<0.001)$.

The impact of the RHD in school functioning domain can be explained by previous findings by White ${ }^{(32)}$, in which it was stated that individuals with RHD may exhibit profound reversible cognitive problems, such as poor attention and concentration.

\section{CONCLUSION}

In conclusion, rheumatic heart disease imposed a considerable burden on the quality of life of the Egyptian pediatric population. In our study, we found that Egyptian patients with rheumatic heart disease had significant impairments on the physical, emotional, social, and school functioning, as compared to their counterpart healthy population. This finding is very important as it confirms the need for follow up and tracking of the HRQoL among patients with rheumatic heart disease. Patients with a higher number of hospital admission are more likely to have more impairment in HRQoL. Nevertheless, further studies with rigorous design, large sample size, and multiregional cooperation are required.

\section{RECOMMENDATIONS}

- Rheumatic heart disease imposed a considerable burden on the quality of life. Thus, it is recommended to follow up and tracking of the HRQoL among children with rheumatic heart disease.

- Interventions on the improvement of HRQOL that focus on psychological aspects need to be provided to the participants.

- A nursing intervention that reflects the psychological needs of the RHD patients should be developed as well.

\section{REFERENCES}

1. Alqanatish J, Alfadhel A, Albelali A et al. (2019): Acute rheumatic fever diagnosis and management: Review of the global implications of the new revised diagnostic criteria with a focus on Saudi Arabia. J Saudi Heart Assoc., 31:273-81.

2. Kotit S, Said K, ElFaramawy A et al. (2017): Prevalence and prognostic value of echocardiographic screening for rheumatic heart disease. Open Heart, 4(2): 702-5.

3. Hofmann S, Wu J, Boettcher $\mathbf{H}$ (2014): Effect of cognitivebehavioral therapy for anxiety disorders on quality of life: A 
meta-analysis. Journal of Consulting and Clinical Psychology, 82: 375-391.

4. Lloyd-Jones D, Hong Y, Labarthe D et al. (2010): Defining and setting national goals for cardiovascular health promotion and disease reduction: the American HeartAssociation's strategic Impact Goal through 2020 and beyond. Circulation, 121:586-613.

5. Rumsfeld J, Alexander K, Goff $D$ et al. (2013): Cardiovascular health: the importance of measuring patient-reported health status: a scientific statement from the American Heart Association. Circulation, 127:2233-49.

6. Kluetz P, Slagle A, Papadopoulos E et al. (2016): Focusing on Core Patient-Reported Outcomes in Cancer Clinical Trials: Symptomatic Adverse Events, Physical Function, and DiseaseRelated Symptoms. Clin Cancer Res., 22:1553-56.

7. Murray C, Vos T, Lozano R et al. (2012): Disability-adjusted life years (DALYs) for 291 diseases and injuries in 21 regions, 1990-2010: A systematic analysis for the Global Burden of Disease Study 2010. Lancet, 380:2197-223.

8. Tóthová V, Bártlová S, Dolák F et al. (2014): Quality of life in patients with chronic diseases. Neuro Endocrinol Lett., 35:11-18.

9. Bradley-Hewitt T, Dantin A, Ploutz M et al. (2016): The impact of echocardiographic screening for rheumatic heart disease on patient quality of life. The Journal of Pediatrics, 175: 123-129.

10. Scheel A, Beaton A, Okello E et al. (2018): The impact of a peer support group for children with rheumatic heart disease in Uganda. Patient Education and Counseling, 101(1): 119-123.

11. Gewitz M, Baltimore R, Tani L et al. (2015): Revision of the Jones criteria for the diagnosis of acute rheumatic fever in the era of Doppler echocardiography a scientific statement from the American heart association. Circulation, 131:1806-18.

12. World Health Organization (1984): Constitution of the World Health Organization basic document. Geneva (Switzerland).http://apps.who.int/bookorders.

13. Varni J, Burwinkle T, Katz E et al. (2002): The PedsQLTM in pediatric cancer: Reliability and validity of the Pediatric Quality of Life InventoryTM Generic Core Scales, Multidimensional Fatigue Scale, and Cancer Module. Cancer, 94:2090-106.

14. Noubiap J, Agbor V, Bigna J et al. (2019): Prevalence and progression of rheumatic heart disease: a global systematic review and meta-analysis of population-based echocardiographic studies. Sci Rep., 9: 17022-26.

15. Hajar R (2016): Rheumatic fever and rheumatic heart disease a historical perspective. Hear Views, 17:120-123.

16.Paar J, Berrios N, Rose J et al. (2010): Prevalence of Rheumatic Heart Disease in Children and Young Adults in Nicaragua. Am J Cardiol., 105:1809-14.
17. Animasahun B, Wobo A, Itiola A et al. (2018): The burden of rheumatic heart disease among children in Lagos: How are we fairing? Pan Afr Med J., 29:150-55.

18. Boyarchuk O, Komorovsky R, Kovalchuk $T$ et al. (2018): Socio-demographic and medical predictors of rheumatic heart disease in a low-risk population. Pediatr Pol., 93:325-30.

19. Borges F, Barbosa M, Borges $R$ et al. (2005): Clinical and demographic characteristics of 99 episodes of rheumatic fever in an acre, the Brazilian Amazon. Arq Bras Cardiol., 84:111-4.

20.Sharma P, Shakya U, Shrestha M (2016): Clinical Profile and Management in Children with Rheumatic Heart Disease in a Tertiary Cardiac Care Center of Nepal. Nepal Hear J., 13:33-6.

21. Gölbasi Z, Uçar Ö, Keles T et al. (2002): Increased levels of high sensitive $\mathrm{C}$-reactive protein in patients with chronic rheumatic valve disease: Evidence of ongoing inflammation. Eur J Heart Fail., 4:593-5.

22. Attar A, Marzban P, Moaref A et al. (2018): The association of plasma high-sensitivity C-reactive protein level with rheumatic heart disease: The possible role of inflammation. Indian Heart J., 70:346-9.

23. Shrestha N, Pilgrim T, Karki P et al. (2012): Rheumatic heart disease revisited: Patterns of valvular involvement from a consecutive cohort in eastern Nepal. J Cardiovasc Med., 13:755-9.

24. Manjunath C, Srinivas P, Ravindranath $K$ et al. (2014): Incidence and patterns of valvular heart disease in a tertiary care high-volume cardiac center: A single-center experience. Indian Heart J., 66:320-6.

25. Yanagawa B, Butany J, Verma $S$ (2016): Update on rheumatic heart disease. Curr Opin Cardiol., 31:162-8.

26. Panepinto J, Pajewski N, Foerster L et al. (2008): The performance of the pedsQL generic core scales in children with sickle cell disease. J Pediatr Hematol Oncol., 30:666-73.

27. Riaz A, Hanif M, Khan I et al. (2018): Quality of life in patients with rheumatic heart disease. J Pak Med Assoc., 68:370-5.

28. Arafa M, Zaher S, El-Dowaty A et al. (2008): Quality of life among parents of children with heart disease. Health Qual Life Outcomes, 6: 91-94.

29.Sharma $S$, Verma $S$ (2015): A clinical evaluation of atrial fibrillation in rheumatic heart disease. J Assoc Physicians India, 63:22-5.

30. Essawy M, Bahgat Z, Kassem H (2010): Health-related quality of life of school-age children with Rheumatic Fever. J Egypt Public Health Assoc., 85 (3-4):205-22.

31.Herring M, Puetz T, O'Connor P et al. (2012): Effect of exercise training on depressive symptoms among patients with a chronic illness: A systematic review and meta-analysis of randomized controlled trials. Arch Intern Med., 172:101-11.

32. White C (2001): Cognitive behavioral principles in managing chronic disease. West J Med., 175:338-42. 